\title{
Control of Spatio-Temporal Dynamics of Broad-Area Semiconductor Lasers by Strong Optical Injection
}

\author{
Satoshi Takimoto, Tomokazu Tachikawa, Rui Shogenji, and Junji Ohtsubo, Member, IEEE
}

\begin{abstract}
Control of spatio-temporal dynamics in broad-area semiconductor lasers is investigated numerically under the condition of strong optical injection. Irregular filamentations of the laser emissions are considerably suppressed as the optical injection ratio is increased. At high optical injections ratios, the laser oscillates with a regular structure in the time-resolved near-field pattern. At the same time, the time-averaged near-field pattern at the exit facet exhibits a good top-hat distribution.
\end{abstract}

Index Terms-Broad-area semiconductor lasers, chaos, dynamic filamentations, nonlinear optics, optical injection.

\section{INTRODUCTION}

H IGH semiconductor lasers are promising coherent light sources for industrial applications, since they have much higher electrical-to-optical power conversion efficiencies than other lasers. One kind of high-power semiconductor laser is the broad-area semiconductor laser. Broad-area semiconductor lasers have similar structures as conventional edge-emitting lasers except that the emitting region at the front facet is a broad stripe that is up to several hundred micrometers wide. In such structures, the effects of carrier diffusion and light diffraction in the active region play a critical role in the dynamics. Broad-area semiconductor lasers are unstable light sources due to the spatial dependence of the laser oscillations [1], [2]. While they are capable of emitting high optical powers, they have rather poor beam quality due to instabilities caused by the microscopic dynamics [3]-[5]. Until recently, most research has focused on how to increase the output power of broad-area semiconductor lasers and little attention has been given to the dynamics, which are responsible for the deterioration in the beam quality.

One instability that occurs in the dynamics of broad-area semiconductor lasers is filamentation [3]-[5]. The spatial positions of carrier hole-burning regions fluctuate with time in the laser cavity and this gives rise to local pulsating oscillations with periods of the order of picoseconds. Since the spatial size of these bright spots is of the order of several micrometers, broad-area lasers exhibit rapid spatio-temporal fluctuations. The presence of filamentations greatly deteriorates the beam quality of the laser, including the time-averaged beam profile

Manuscript received October 03, 2008; revised January 08, 2009. First published May 12, 2009; current version published July 15, 2009.

S. Takimoto and T. Tachikawa are with the Graduate School of Engineering, Shizuoka University, Naka-ku, Hamamatsu 432-8561, Japan.

R. Shogenji and J. Ohtsubo are with Faculty of Engineering, Shizuoka University, Naka-ku, Hamamatsu 432-8561, Japan (e-mail: tajohts@ ipc.shizuoka.ac.jp).

Color versions of one or more of the figures in this letter are available online at http://ieeexplore.ieee.org.

Digital Object Identifier 10.1109/LPT.2009.2022181 and laser coherence. In spite of several investigations, the origin of dynamic filamentation in broad-area semiconductor lasers is not currently fully understood. Studies have been conducted on controlling and stabilizing the filamentations in broad-area semiconductor lasers. Optical feedback and optical injection are frequently employed to partially or completely suppress the effects of filamentations [7]-[11]. It is widely known that the dynamics of broad-area semiconductor lasers are strongly affected by optical injection; however, quantitative discussions of this are still required. In particular, the relationship between dynamic filamentations and the static beam profile is very important from the viewpoint of applications.

In this letter, we numerically study the control of filamentations in broad-area semiconductor lasers by optical injection. Using a strong optical injection scheme, irregular filamentations become regular oscillations and the near-field pattern of the laser beam has a clean and periodic profile that resembles that produced by a superposition of higher spatial modes. This is consistent with previous experimental results [7]. The time-averaged top-hat characteristics of the beam at the exit facet of the laser are also evaluated.

\section{THEORY}

Here, we briefly introduce a theoretical model for optical injection in a broad-area semiconductor laser. The model is for an index-guided broad-area laser. Considering the spatial dependence along the stripe width, the laser field $E(x, t)$ ( $x$ is the coordinate along the stripe width) is given by [1]

$$
\begin{aligned}
\frac{\partial E(x, t)}{\partial t}= & i D_{e} \frac{\partial^{2} E(x, t)}{\partial x^{2}} \\
& +\frac{1}{2}(1-i \alpha) G_{n}\left\{n(x, t)-n_{\mathrm{th}}\right\} E(x, t) \\
& +\frac{\kappa_{\text {inj }}}{\tau_{\text {in }}} E_{m}(t) \exp (-i \Delta \omega t)+R_{\mathrm{sp}}
\end{aligned}
$$

where $D_{e}=c / 2 k_{0} \eta^{2}$ ( $c$ is the speed of light in vacuum, $k_{0}$ is the wavenumber, and $\eta$ is the refractive index of the medium) is the diffraction coefficient, $\alpha$ is the linewidth enhancement factor, $G_{n}$ is the gain, $n_{\mathrm{th}}$ is the carrier threshold, $\kappa_{\mathrm{inj}}=r_{\mathrm{inj}} / r \sqrt{\left(1-r^{2}\right) / \eta}\left(r_{\mathrm{inj}}\right.$ is the actual injection fraction and $r$ is the reflectivity at the facet for the laser amplitude) is the injection coefficient, $\tau_{\text {in }}$ is the round-trip time of light inside the cavity, $\Delta \omega=\omega_{m}-\omega$ is the frequency difference between the injection laser and the laser under consideration, $R_{\mathrm{sp}}$ is the noise term induced by spontaneous emission, and $E_{m}$ is the optical field of the master injection laser. The spontaneous emission term is included in the rate equation since the actual laser oscillations contain noise effects. However, these have 
little effect on the laser dynamics when the noise induced by spontaneous emission is small. The rate equation is the same as that of a narrow-stripe edge-emitting laser except for the first term on the right-hand side of the equation, which accounts for diffraction in the laser cavity. The filamentation process depends sensitively on $\alpha$, and $\alpha$, in turn, is also affected by dynamic filamentations. However, we assume that $\alpha$ is constant since in a previous study good agreement was obtained between theory and experiment under the assumption of constant $\alpha$ [3].

The carrier density $n$ also has spatial dependence and it is given by [1]

$$
\begin{aligned}
\frac{\partial n(x, t)}{\partial t}=D_{n} \frac{\partial^{2} n(x, t)}{\partial x^{2}} & +\frac{J}{e d}-\frac{n(x, t)}{\tau_{s}} \\
& -G_{n}\left\{n(x, t)-n_{0}\right\}|E(x, t)|^{2}
\end{aligned}
$$

where $D_{n}=l_{d}^{2} / \tau_{s}\left(l_{d}\right.$ is the diffusion length) is the diffusion coefficient, $J$ is the injection current, $e$ is the elemental charge, $d$ is the thickness of the active layer, $\tau_{s}$ is the carrier relaxation time, and $n_{0}$ is the carrier density at transparency. The first term on the right-hand side of (2) represents carrier diffusion. The laser displays a rich variety of dynamics on a microscopic scale due to the presence of the diffraction coefficient in the field equation and the diffusion coefficient in the carrier-density equation.

\section{NUMERICAL RESULTS}

We employ a simple difference method to numerically solve the rate equations. Since the equations include the dimension corresponding to the optical wavelength, the calculation must be performed on a time scale much smaller than a femtosecond. The spatial step for the calculations is chosen to be much smaller than the optical wavelength. In this numerical simulation, for simplicity, we assume that the stripe width of the active layer is $w=50 \mu \mathrm{m}$ and that the continuous-wave injection beam is rectangular with a width of $40 \mu \mathrm{m}$. The beam is symmetrically injected relative to the center of the active layer in the broad-area laser. We used the following values for parameters in the numerical calculations: cavity length $l=500 \mu \mathrm{m}$, optical wavelength $\lambda=0.780 \times 10^{-6} \mathrm{~m}, \Delta \omega / 2 \pi=0 \mathrm{GHz}, r=0.1$, $\alpha=3.0, G_{n}=2.0 \times 10^{-13} \mathrm{~m}^{3} / \mathrm{s}, n_{\mathrm{th}}=5.11 \times 10^{24} \mathrm{~m}^{-3}$, $n_{0}=1.30 \times 10^{24} \mathrm{~m}^{-3}, \tau_{s}=3.0 \times 10^{-9} \mathrm{~s}, l_{d}=3.0 \times 10^{-6} \mathrm{~m}$, $D_{e}=1.44 \mathrm{~m}^{2} / \mathrm{s}, D_{n}=3.00 \times 10^{-3} \mathrm{~m}^{2} / \mathrm{s}, d=0.1 \times 10^{-6} \mathrm{~m}$, and $\eta=3.59$.

Fig. 1 shows the results for dynamic filamentations at several optical injection ratios with a fixed bias injection current of $1.5 J_{\mathrm{th}}$, where $J_{\mathrm{th}}$ is the threshold current. The injection ratio is defined as $r_{\text {inj }}=\left|E_{\text {inj }}\right| /\left|E_{0}\right| . E_{0}$ is the output amplitude of the broad-area laser at the bias injection current. Fig. 1(a) shows a filament oscillation that occurs between 29.5 and 30.0 ns (vertical axis) for the free-running state. The horizontal axis corresponds to the exit facet of the broad-area laser. Bright spots propagate irregularly in a zigzag manner as a function of time. The typical spatial spot size calculated from the pattern correlation is about $10 \mu \mathrm{m}$ and the temporal filament duration is less than 30 ps. The behaviors of filaments and the filament sizes agree well with the results of previous experiments [3]. Fig. 1(b) is the result for an injection ratio of $r_{\text {inj }}=0.4$. The laser exhibits irregular oscillations, but the filament behavior differs from that
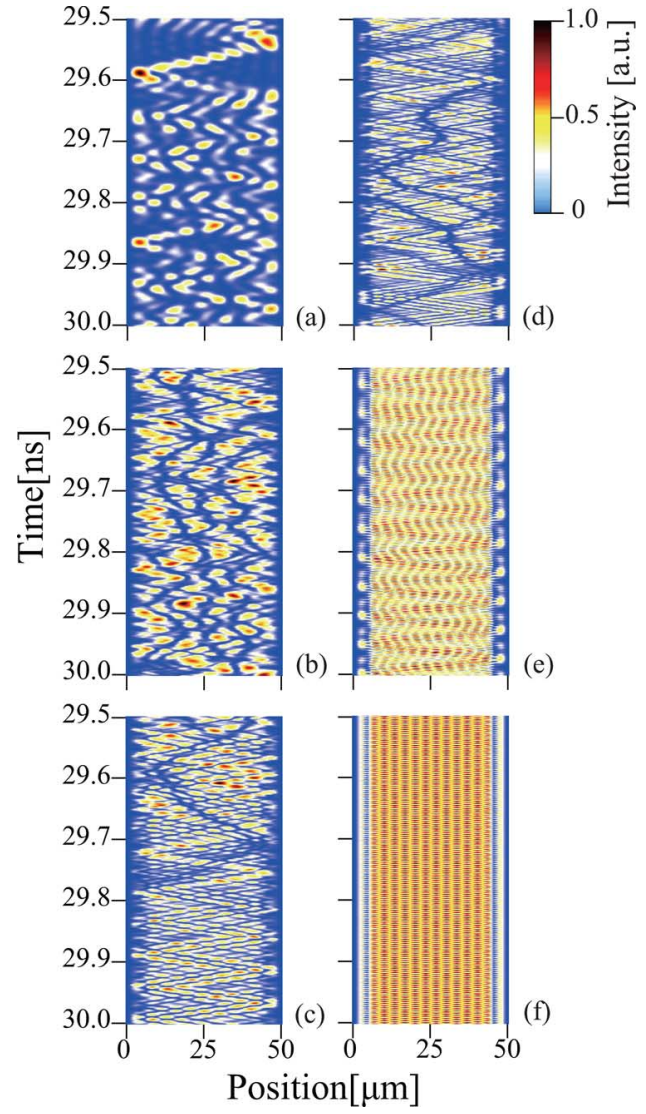

Fig. 1. Near-field patterns for various optical injections at bias injection current of $1.5 J_{\mathrm{th}}$ : (a) no optical injection; (b) $r_{\mathrm{inj}}=0.4$; (c) $r_{\mathrm{inj}}=0.8$; (d) $r_{\mathrm{inj}}=$ $1.2 ;$ (e) $r_{\text {inj }}=1.6$; and (f) $r_{\text {inj }}=2.0$.

of a solitary oscillation and the temporal duration of the filament is reduced. In Fig. 1(c) and (d), which are for optical injection ratios of $r_{\mathrm{inj}}=0.8$ and 1.2, respectively, the laser still undergoes irregular oscillations, but some regular structure can be seen in the pattern and the filament durations are shorter. For an optical injection ratio of $r_{\mathrm{inj}}=1.6$, the pattern begins to exhibit a remarkable periodic structure as shown in Fig. 1(e), there are still filament oscillations. For strong optical injection of $r_{\text {inj }}=2.0$ in Fig. 1(f), the laser oscillates with a periodic structure not only temporally but also spatially. Due to the space limitations, we cannot show the spectrum analysis results for the obtained filament patterns; however, remarkable high-frequency components are observable at higher optical injections. Detailed analysis will be given in another paper.

The time-averaged near-field patterns were calculated to demonstrate the macroscopic beam quality. Fig. 2 shows the result for the intensity profile along the stripe width. Since we assumed a rather low bias injection current close to the threshold and an index-guided structure, for single oscillations the laser beam typically has two large peaks at both edges of the stripe, which was also observed in the experiment [12]. When the optical injection ratio is increased, the side peak intensities are suppressed and the laser beam has a flat top-hat profile, as shown in Fig. 2(b) and (c). Such flat top-hat beam shapes are very important for practical applications. However, the flat-top beam profile collapses and spatial periodicity is 


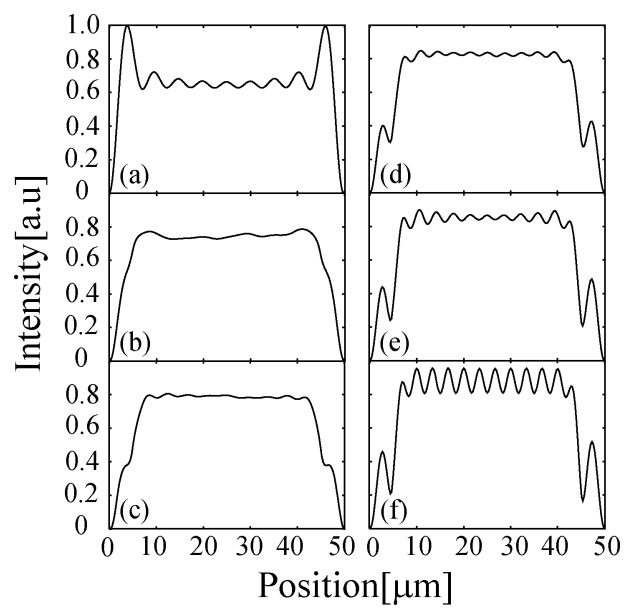

Fig. 2. Time averaged near-field patterns corresponding to Fig. 1.

enhanced with a further increase in the optical injection, as shown in Fig. 2(d) to (f) in accordance with the periodic spatial filamentations in Fig. 1. The peak intensities at both edges of the stripe outside of the optical injection areas increase with an increase in the injection ratio. As the optical injection ratio is increased, the number of low-intensity peaks within the optically injected area increases, since the intensity inside the optical cavity increases. The number of excited spatial modes increases with an increase in the bias injection current. The number of excited spatial modes in broad-area semiconductor lasers for bias injection current variations has been explained by the superposition of Hermite-Gaussian polynomials [12].

\section{CONCLUSION}

We have demonstrated that filamentations in broad-area semiconductor lasers are moderately suppressed by strong optical injection and the quality of the time-averaged beam profile is appreciably improved. One of the reasons for this improvement may be the suppression of carrier diffusion due to strong and uniform optical injection. However, the origins of filament suppression and beam shaping are left for further study. In this present study, we only considered the case of zero detuning between the broad-area semiconductor laser and the injection laser. The dynamics in the presence of a finite detuning are also left for further study. In the present study, we assumed that optical injection was achieved using a square beam. Such a configuration is not difficult to achieve by employing some optical elements and filters. However, there are several schemes for optical injection. A more realistic beam for optical injection is a beam with a smooth profile (e.g., a Gaussian beam). We have performed simulations using such beams and found that a larger injection ratio is required to obtain a periodic filament structure than with a square beam. However, similar trends in the development of dynamic filamentations were obtained. Since a broad-area laser has a wider stripe, the positioning of the injection beam at the active layer is not so critical. Also, adjusting the beam position on a micrometer scale is not difficult by employing the current precision technique.

Finally, we point out the achievability of the injection strength used in the numerical simulations. In the simulations, we assumed a strong optical injection with an amplitude ratio as large as $r_{\mathrm{inj}}=2.0$, which is four times greater than the output power of a single laser oscillation. For broad-area semiconductor lasers, the maximum possible injection current is usually several times larger than the threshold current, although the maximum injection current varies strongly depends between lasers. For example, a bias injection current of up to ten times the threshold was experimentally demonstrated in a broad-area semiconductor laser [12]. Therefore, as long as the laser is biased at a relatively low injection current, we can avoid damaging the laser by strong optical injection (e.g., causing catastrophic optical damage).

\section{REFERENCES}

[1] J. Ohtsubo, Semiconductor Lasers: Stability, Instability and Chaos, 2nd ed. Berlin, Germany: Springer-Verlag, 2007.

[2] E. Gehrig and O. Hess, Spatio-Temporal Dynamics and Quantum Fluctuations in Semiconductor Lasers. Heidelberg, Germany: SpringerVerlag, 2003.

[3] I. Fischer, O. Hess, W. Elsäßer, and E. Göbel, "Complex spatio-temporal dynamics in the near-field of broad-area semiconductor laser," EuroPhys. Lett., vol. 35, pp. 579-584, 1996.

[4] O. Hess and T. Kuhn, "Maxwell-Bloch equations for spatially inhomogeneous semiconductor lasers. II. Spatiotemporal dynamics," Phys. Rev. A, vol. 54, pp. 3360-3368, 1996.

[5] T. Burkhard, M. O. Ziegler, I. Fischer, and W. Elsäßer, "Spatio-temporal dynamics of broad area semiconductor lasers and its characterization," Chaos Solitons Fractals, vol. 10, pp. 845-850, 1999.

[6] J. Kaiser, I. Fischer, W. Elsäßer, E. Gehrig, and O. Hess, "Mode-locking in broad-area semiconductor lasers enhanced by picosecond-pulse injection," IEEE J. Sel. Topics Quantum Electron., vol. 10, no. 5, pp. 968-973, Sep./Oct. 2004.

[7] P. D. van Voorst, H. L. Offerhaus, and K.-J. Boller, "Single-frequency operation of a broad-area laser diode by injection locking of a complex spatial mode via a double phase conjugate mirror," Opt. Lett., vol. 31, pp. 1061-1063, 2006.

[8] S. K. Mandre, I. Fischer, and W. Elsäßer, "Control of the spatiotemporal emission of a broad-area semiconductor laser by spatially filtered feedback," Opt. Lett., vol. 28, pp. 1135-1137, 2003.

[9] Y. Fujita and J. Ohtsubo, "Optical-feedback-induced stability and instability in broad-area semiconductor lasers," Appl. Phys. Lett., vol. 87, pp. 031112-1-3, 2005.

[10] S. K. Mandre, I. Fischer, and W. Elsäßer, "Spatiotemporal emission dynamics of a broad-area semiconductor laser in an external cavity: Stabilization and feedback-induced instabilities," Opt. Commun., vol. 244, pp. 355-365, 2005.

[11] N. Gaciu, E. Gehring, and O. Hess, "Control of broad-area laser dynamics with delayed optical feedback," in Handbook of Chaos Control, E. Scholl and H. G. Schuster, Eds. Weinheim: Wiley-VHC, 2008, ch. 20.

[12] T. Asatsuma, Y. Takiguchi, S. Frederico, A. Furukawa, and S. Hirata, "Successive phase change and stability of near-field patterns for broadarea laser diodes," Proc. SPIE, vol. 6104, p. 61040C, 2006. 